\title{
EL DOLOR DE LOS PACIENTES AYMARA DE LA COMUNA DE PUTRE: PRÁCTICAS TERAPÉUTICAS Y POLÍTICAS DE SALUD INTERCULTURAL
}

\author{
PAIN IN AYMARA'S PATIENTS OF PUTRE: THERAPEUTICS PRACTICES \\ AND POLITICS OF INTERCULTURAL HEALTH
}

\begin{abstract}
Serena Caroselli*
Desde antropología médica, como enfoque, se analiza cómo en el Centro de Salud Familiar de Putre (CESFAM) se atienden los pacientes con dolor. El trabajo de investigación que se llevó a cabo durante siete meses -septiembre de 2011 hasta febrero de 2012- permite abordar analíticamente casos de pacientes del poblado de Putre y de las localidades de precordillera y cordillera de la comuna del mismo nombre y como ellos, ante los saberes médicos plurales, hablan de dolor y lo enfrentan. Aunque el Programa Especial de Salud de los Pueblos Indígenas (PESPI) incluye médicos aymara en el equipo de trabajo del CESFAM, cada etnomedicina maneja el dolor en su complejidad desde su perspectiva profesional en el campo de la salud intercultural, transformando a través de las prácticas terapéuticas plurales esa ambigüedad, que el dolor genera, en la vida del sujeto que sufre.

El dolor del paciente aymara se incluye en el campo de la salud intercultural, pero la condición íntima y privada del sujeto se hace pública y política cuando se maneja a nivel institucional.

Palabras claves: dolor, salud intercultural, prácticas terapéuticas integradas.

Through the medical anthropology's perspective we analyze how are attended patients with pain by the Center of familiar health of Putre (CESFAM). The field research, performed from September of 2011 to February of 2012, allowed to us to analyze the cases about patients of Putre and other about patients of pre cordillera's and cordillera's villages. They speak about pain and used to take care about it moving around different medical knowledge. The especial program of native's populations includes aymara's therapists in the CESFAM's team. Every etnomedicine manages pain in its complexity from its professional perspective inside the intercultural health's field, transforming trough the therapeutics and plurals practices this ambiguity that pain causes in the life of the subject that suffer. Pain of aymara's patients stays inside the intercultural health's field, the private and intimate condition became public and politic when it's managed from an institutional position.
\end{abstract}

Key words: pain, intercultural health, therapeutic and integrates practices.

\section{Introducción}

Cuando hablamos de dolor siempre resulta difícil entender, en términos específicos, lo que significa para los pacientes que hablan de su malestar. Por esta razón necesitamos referirnos a las raíces etimológicas de nuestros idiomas que desde el latín nos permiten encontrar el sentido de nuestro lenguaje, el mismo que utilizamos para describir lo que pasa cuando nos enfermamos. En italiano como en castellano la palabra "dolor" nace de la raíz Dol-éo (Devoto 1995) que indica algo que se nos quiebra adentro. El dolor surge cuando algo queda quebrado en nuestro cuerpo y en nuestra vida. Para referirnos al dolor muchas veces hablamos en términos de malestar, sufrimiento, padecimiento y la palabra "sufrir" etimológicamente se refiere a Sub-ferre (Devoto 1995) "conllevar", "aguantar".
Esta diferencia entre estas dos palabras, las que los pacientes utilizan cuando hablan de su condición de sufrimiento, define una separación que la biomedicina siempre adopta. El dolor es algo físico, en cambio el sufrimiento se refiere a cómo nos sentimos. $\mathrm{La}$ antropología médica critica el pensamiento maniqueo que opera una separación entre alma y cuerpo, y se refiere al cuerpo y a la enfermedad en términos de incorporación por parte del sujeto. En lo que atañe al dolor resulta más difícil analizar lo que es visto que solamente quien vive el dolor sabe cómo se manifiesta y no puede transmitirlo tal cómo lo vive, ya que este destruye el lenguaje y el mundo de la persona que lo padece (Scarry 1985: 40).

Uso esta premisa lingüística porque cuando hablamos del paciente aymara, aunque la mayoría de las personas de la región hable también el castellano, el idioma que se utiliza para expresar

\footnotetext{
* Università degli studi di Roma “Sapienza”, Roma, Italia. Correo electrónico: serena.caroselli@libero.it
} 
el dolor es el aymara y, a lo largo de mi estancia en el campo, muchas veces los terapeutas aymaras hablaban con sus pacientes en su idioma, y los adultos mayores de la zona de cordillera utilizaban el aymara con los médicos del CESFAM. El diálogo entre los dos actores se complicaba mucho en la medida en que el profesional de salud manejaba casos cada vez más complejos, donde el diálogo resultaba ser fundamental para brindar una atención de calidad. Cada una de las personas con dolor ocupa su propia narración para expresar lo que tiene adentro, y entre los aymaras del norte de Chile, los que viven en una zona rural, el elemento cultural y propio de la tradición aymara le otorga al dolor ciertas coordenadas de significación dirigidas a contenerlo, explicarlo y a enfrentar las prácticas de curación de una forma que, sin embargo, resulta desconocida a los profesionales de salud que operan en los dispositivos médicos rurales. En aymara no existe una palabra que corresponda al término "dolor", existen discursos que explican y significan el dolor vivido. Cada vez que iba a preguntar a los pacientes sobre su dolor, el término que solían usar era Usu, literalmente "enfermedad", mientras que al enfermo se le dice Usutua. Cuando el yatiri ${ }^{1}$ les preguntaba a sus pacientes sobre cómo se sentían, utilizaba esta fórmula: ¿Kuna Ustama?, es decir, "¿Qué te duele?"

En la etapa inicial de mi investigación quise hallar una traducción literal del término "dolor", intentando un acercamiento comparativo y buscando en el diccionario aymara-castellano/castellanoaymara algunas fórmulas que pudieran referirse a este tema, sin lograr descubrir una palabra específica que tuviera que ver con el dolor: "iyawa es la interjección de aceptación de dolor; atataw/atatay la interjección que expresa dolor" (García Choque 1997: 52) Parece entonces no existir un sinónimo de la locución que nos interesa y, cómo el idioma aymara es aglutinativo y las locuciones se acoplan en la creación de fórmulas que incorporan el sentido complejo de la palabra, usutatwa nace muy probablemente de la fusión de usuta-atataw, que atañe al sentirse mal en relación con el dolor padecido, y podría ser también que esto incluya una aceptación del dolor mismo. Para respaldar este acercamiento a las formas de "hablar" la condición dramática del dolor, voy a recurrir a un fragmento de una entrevista para rematar la idea según la cual hablar de dolor resulta muy complicado. De hecho, tanto la salud como la enfermedad -y por lo tanto la condición de dolor-representan no sólo algo biológico, sino algo cultural reproducido y significado desde un punto de vista social. En este sentido cualquier discurso sobre el dolor, pese al contexto en que se dé, resulta ser inadecuado para expresar el significado profundo de la condición del sujeto que vive y percibe el dolor a lo largo su propia vida.

En aymara hablar de dolor implica una narración que pueda explicar el sentido que adquiere para la persona que lo padece; las narrativas se caracterizan por ser fundamentales en la delimitación de las esferas de manifestación del dolor mismo, gracias a las expresiones idiomáticas que cobran un significado específico para cada persona que sufre esta situación.

S: ¿Don G. No me acuerdo, ¿cómo se dice "dolor" en aymara?

G: Usutua se dice. "Me duele" se dice usutua, cómo chuyma usutua. Usutatwa, "me duele, cómo me siento enfermo".

$\mathrm{S}: ~ ¿ \mathrm{O}$ sea, me siento enfermo, en toda mi persona?

G: Sí, exactamente, usutatwa "me siento enfermo".

$\mathrm{S}$ : ¿Entonces hay varias formas de decir "dolor"?

G: Sí, usutua "me duele", usutatwa "me siento enfermo, estoy enfermo".

S: Es muy interesante, pero usted me dice usutua y usutatwa ¿Las dos palabras se utilizan para hablar de dolores distintos o no? G: Usutua como para referirse al hecho que me duele la rodilla, y usutatwa para decir "me siento enfermo, estoy enfermo". S: ¿Tienen la misma raíz entonces?

G: Sí, si te duele estás enfermo.

$\mathrm{S}$ : Pero igual puede existir un dolor pasajero, y un dolor...

G: ...un dolor permanente, eso se dice en aymara usutatwa, bueno en castellano se dice: "me duele siempre, me duele, no se me pasa el dolor".

S: ¿Y el dolor permanente puede ser como sufrimiento?

G: Sí, de este dolor, como de pena pero no solo, no sólo que me siento triste, penoso, angustiado, que tengo una angustia.

S: ¿Hay varias formas entonces de decirlo, con respecto a la tipología de dolor que uno percibe? 
G: Sí, las personas no saben su idioma, se olvidan, hay un dolor más grande, afuera de la tristeza, o del dolor de las rodillas, es uno, y se te pone adentro en todo tu ser persona. Para hablar hay que meditar. Hay que sacar de la mente lo que significa para decirlo².

En este entorno indígena, así como en cada contexto de significación del dolor, sobresale cómo desaparezca nuestra capacidad de definir exactamente lo que es el dolor. Frente a la afirmación de nuestro actor notamos que tampoco las categorías émicas para nombrar el sentido complejo de dolor parecen adecuadas para la comprensión.

Sin embargo, cuando hablamos de dolor surgen las contradicciones de la cultura biomédica monolítica; esta suele diferenciar la esfera de la percepción del paciente (respecto de un malestar) y la del diagnóstico clínico, ambas necesarias para enfrentar las terapias. De todas maneras, también la medicina tradicional construye y de-construye significados sobre el dolor, nombrados de una forma familiar y determinados culturalmente, que sin embargo quedan abiertos a la posibilidad de ser transformados; en este sentido la medicalización de la vida de los pacientes indígenas genera la transformación de las categorías que el mismo sistema biomédico domina para designar y devolverle sentido al padecimiento.

Cuando hablamos de dolor necesitamos evaluar la complejidad de la reflexión porque "él tiene tres polaridades: universalidad, individualidad, sociabilidad", como afirma Le Breton (Le Breton 1995).

Si nos detenemos sobre el significado del dolor y sobre la experiencia que hace la persona que sufre, las categorías biomédicas, que valoran las evidencias del cuerpo para hacer un diagnóstico, se hacen críticas a la hora de emprender el tratamiento del paciente aymara; él hablando de su condición recurre a modelos explicativos propios de su cosmovisión, remite las causas de su sufrimiento a significados no compartidos con los profesionales.

Porque: "el dolor está integrado a una visión del mundo, en todas las sociedades, ésta atribuye significados, elabora medidas simbólicas y prácticas dirigidas a disminuir su capacidad de destrucción" (Cozzi 2012: 94, trad. mía.). El dolor expresado físicamente en la vida cotidiana está incorporado por la persona, y conlleva, en el caso del paciente aymara, una herida abierta en el cuerpo a consecuencia de una historia de violencia, que sigue actuando en la vida del sujeto.

La violencia de la que hablan los pacientes entrevistados nace en relación con la historia colectiva del grupo aymara de Chile, al proceso de chilenización, es decir, la inclusión de las personas indígenas dentro del Estado Nacional Chileno, y a las consecuencias de una pérdida de identidad en cuanto grupo indígena: identidad como concepto dinámico y sensible a ser transformado a lo largo del tiempo.

Ellos, conscientes de una herida que nunca sana, expresan, a través de sus narrativas, el drama de la historia del pueblo aymara: en relación con este fenómeno existe el hábito de hablar en términos de dolor, una palabra usada bastante asiduamente. El dolor es una metáfora que surge en los discursos más allá de la evidencia de una enfermedad diagnosticada, y representa un síntoma de malestar dependiente también de la historia de desolación y de violencia pasada. En los casos de los pacientes entrevistados está relacionado con el llevar una vida de soledad, donde muchas veces esto depende de la migración de los aymaras, sobre todo de los jóvenes, hacia la ciudad por razones de trabajo y estudio. De hecho la mayoría de los pacientes de la cordillera son adultos mayores, en mínima parte adultos, y todos están afectados por patologías dependientes del envejecimiento de la población, cuales son patologías reumáticas, degenerativas y, en algunos casos, crónico respiratorias.

Estamos de acuerdo con que la presencia de la biomedicina en las zonas rurales haya solucionado muchos problemas asociados a enfermedades infectivas, aunque hoy no logre resolver sola todas las patologías. Entre estas, la mayoría dependen de una expectativa de vida más alta, aunque de menor calidad. Cada vez más el paciente indígena se enferma de las patologías dichas de la modernidad ${ }^{3}$, que dependen de los cambios climáticos, de la presencia de la contaminación ambiental, y del cambio de alimentación. Estos factores epidemiológicos nos ayudan a tener claro el perfil de nuestros actores; ellos pueden recibir, en las zonas rurales del norte de Chile, un atención médica intercultural fomentada desde el Servicio de Salud de Arica, junto con la Municipalidad de Putre. Esto genera la posibilidad para ellos de atenderse con el servicio médico integrado que nace de la inclusión de los terapeutas aymara en el equipo del CESFAM. Observando las dinámicas que caracterizan las políticas de la salud intercultural 
se distinguen tres actores institucionales: el Servicio de Salud, con el programa PESPI, la municipalidad y los dirigentes aymara de la comuna. No podemos dejar de razonar sobre lo que pasa cuando el sistema médico está muy involucrado en las lógicas políticas. Muchas veces los dispositivos de salud responden a estrategias hechas para fortalecer el control del sujeto indígena, a través del manejo de su salud. La medicalización extrema -también ahí donde la comunidad tiene su sistema médico-, el suministro de medicamentos -aunque en muchos casos no sirva-, el crecimiento de las visitas obligadas impiden la mejoría de la existencia del paciente indígena; esta nace también de la capacidad del profesional de hacerse cargo de la condición del enfermo y, aún más en el caso específico del paciente que vive al margen, desde el momento que su vida está cargada por la presencia de dolor.

Antes de profundizar algunos casos para fundamentar este estudio antropológico a través de la investigación etnográfica, hay que reflexionar y puntualizar sobre la concreción de las acciones médicas observadas, de las políticas vigentes y de las maneras de manejar el paciente con dolor. Él puede atenderse con diferentes figuras profesionales, incluidos los terapeutas aymaras que están contratados oficialmente por el CESFAM. Esta situación favorece la atención básica, aunque medicalice, en la mayoría de los casos, la vida de la comunidad de Putre, y carece cada vez más de atención específica a personas con dolor crónico las que necesitarían espacios distintos de atención. El paciente se atiende a la vez con el equipo biomédico y con el equipo intercultural para manejar la condición de invalidez que, en la mayoría de los casos, el dolor provoca.

\section{El sistema intercultural de atención en salud}

Para enmarcar este nuevo campo que es la salud intercultural, entendiendo por campo un "espacio social donde actúan instituciones específicas, reguladas por relaciones de fuerza dentro de las cuales operan los actores que lo habitan (Bourdieu 2003)", hay que definir como a nivel mundial, después de la conferencia de Alma Ata ${ }^{4}$ en 1978, los países que asistieron establecieron una estrategia de salud global; según esta estrategia se implementaba la atención médica como herramienta fundamental para alcanzar el objetivo de una superación de las iniquidades en la atención a nivel mundial. A través de algunas etapas cronológicas la salud se incluyó entre los derechos fundamentales; antes en la Declaración de Rio de 1992, después de un año en la Conferencia Mundial de los Derechos Humanos nació el Foro Permanente de los Pueblos Originarios. En un principio el tema de la interculturalidad quedaba bastante incierto, hasta el 2001, desde cuando está vigente el Convenio 169 de la OIT (Organización Internacional de Trabajo).

El primer momento en el cual se habló de interculturalidad en el marco de las políticas mundiales fue cuando la Organzacion mundial de la salud (OMS) y la Organización panamericana de la salud (OPS) definieron los objetivos la "década de los pueblos originarios”, entre 1994 y 2004. Los países latinoamericanos miembros tomaron en cuenta los problemas y las soluciones en la cuestión indígena desde una perspectiva intercultural: "En esta ocasión la OPS propuso la inclusión de una perspectiva intercultural en el campo de la salud como estrategia necesaria para lograr la equidad de atención y un servicio médico de calidad para las poblaciones de América y, más en lo específico, para las poblaciones indígenas" (Ramírez Hita, 2011: 43).

Respecto de Chile, un primer diálogo intercultural se dio en 2001 cuando se adoptó el Convenio 169 y nació el Programa Orígenes para el fomento y el desarrollo de los pueblos originarios, con respeto a las diferencias culturales, con el objetivo de una cooperación entre Estado y ciudadanos chilenos.

La política intercultural miraba a constituir el desarrollo y la mejoría de la calidad de vida de los pueblos originarios, aymara, atacameño, mapuche, de los sectores urbanos y también rurales. Se implementó un modelo intercultural a través del fortalecimiento de la medicina aymara en la región $\mathrm{XV}$, sensibilizando a los funcionarios públicos acerca de los asuntos indígenas; elementos para mejorar las relaciones entre profesionales y pacientes, a través de la inclusión del concepto de pertinencia cultural en la atención médica.

En este sentido se reconfiguró el espacio terapéutico desde un punto de vista teórico; esto generó lo que Guillaume Bruno Boccara (2007) llama subcampo de la salud intercultural, incluido dentro de un campo burocrático más amplio (lo de la salud pública chilena) tanto desde un punto de vista institucional como en el aspecto administrativo.

Este análisis desvela cómo las retóricas interculturales, dentro del Estado chileno, parecen reproducir relaciones de poder: 
"Planteamos que la salud intercultural representa una nueva ingeniería social que se inscribe dentro de un nuevo diagrama de saber/poder o de una gubernamentabilidad de un nuevo tipo, que tiende a producir nuevos sujetos étnicos, colectivos e individuales a través de una doble dinámica de etnicización y de responsabilización. A través de la puesta en marcha de programas de salud intercultural, son las relaciones entre cuerpo social y cuerpo individual, esfera pública y esfera privada, individuo y población y Estado y ciudadano que se encuentran repensadas y modificadas [...] Lo que está en juego en la delimitación de este nuevo segmento de esfera de intervención del Estado es mucho más que el respeto a la alteridad o el reconocimiento a la diversidad cultural del país (Boccara 2007)".

Aquí retomamos la idea de etnogubernamentabilidad para referirnos a las formas de control de la población indígena que, respecto del tema de la salud, generan la medicalización del espacio terapéutico, donde los problemas reales se hacen invisibles a través de la instrumentalización del factor cultural. Las políticas orientan la población a elegir y desear un modelo hegemónico que instrumentaliza el tema indígena: "la predisposición a reducir la interculturalidad como algo cultural desvela los objetivos de asimilación e integración, y en particular modo invisibilizar los procesos socioeconómicos que reducen o impiden el nacimiento de relaciones interculturales respetuosas y équas (Menéndez 2006: 61)".

En la comuna de Putre, dentro del Centro de Salud Familiar, a los usuarios que acuden se les brinda teóricamente un modelo intercultural transterapéutico y plural como respuesta a la heterogeneidad de contexto de atención médica. La solución adoptada fue la de incluir e implementar la presencia de terapeutas aymaras en el consultorio, para realizar un trabajo junto con la plantilla de los profesionales.

El pluralismo médico presente en el territorio está reconocido oficialmente a través del modelo de salud intercultural, y se traduce en la valorización de las prácticas terapéuticas indígenas como útil dentro del consultorio donde se atienden los pacientes. Este patrón, aun basándose en la adopción de ciertas medidas del Convenio 169, sin embargo muchas veces no se concretiza en una acción terapéutica integrada. Especialmente en la gestión del dolor de los pacientes indígenas surge cierta incompatibilidad entre discursos y prácticas de la interculturalidad. De hecho, el servicio del CESFAM no destruye las barreras culturales que le impiden al paciente aymara con dolor de tener una atención médica de calidad. Los problemas de salud de la comunidad muchas veces se ocultan a través de la instrumentalización del factor cultural, se despolitizan los problemas sanitarios culturalizando las soluciones: con ello me refiero específicamente a la contratación de terapeutas aymara dentro de los centros de salud. Este es un factor que sirve a menudo para mitigar la insatisfacción de la comunidad frente a un servicio médico de baja calidad, sobre todo desde un punto de vista técnico.

Esta falta de gestión integrada entre los dos saberes médicos se hace más evidente a la hora de observar casos de pacientes que padecen una condición de dolor agudo o crónico. Pese a los éxitos indiscutibles alcanzados por la biomedicina en lo que concierne por ejemplo a las enfermedades infecciosas, no resulta cumplir con las demandas de atención por parte de los pacientes que padecen un dolor crónico. De hecho, ellos requieren (véase las entrevistas más abajo) de alguien que sepa hacerse cargo de su condición, que comparta con él y con su familia la dificultad que deriva de esta situación de padecimiento; porque el dolor amenaza la capacidad humana de manejarlo física y existencialmente y un enfoque exclusivamente clínico genera la incapacidad de satisfacer las necesidades de los pacientes. Al acercarse a los pacientes, los terapeutas aymaras asumen el límite de sus propias prácticas, sobre todo cuando experimentan la imposibilidad de disminuir el dolor de una forma inmediata, así como sucede a través del suministro de fármacos, gracias a su poder analgésico. Sin embargo, las plantas medicinales que ocupan tienen un "efecto acumulativo" sumamente valorado por los pacientes que las usan junto con sus terapeutas, pero también en las prácticas de autoatención.

Estas distintas visiones terapéuticas, aunque las dos sean necesarias, entran en crisis frente al dolor que parece representar la condición límite para la persona, una experiencia que está llena de ambigüedad y complejidad. Cada una de las dos perspectivas médicas responden a paradigmas que, aunque no compatibles desde un punto de vista 
epistemológico, pueden confluir en las prácticas de atención y llegar a ser, de una forma integrada, más eficientes para el paciente.

Concretamente, lo que ocurre en el Centro de Salud Familiar responde a una separación entre prácticas médicas por lo cual cada enfoque orienta la forma de manejar el dolor con sus propias herramientas y remedios, desatendiendo por ende una colaboración posible y fecunda.

El Centro de Salud Familiar de Putre fue reconocido como dispositivo familiar intercultural de atención a la comunidad solo en 2009. En un principio solo había una posta rural que, en 1996, fue convertida en un consultorio general. La posta rural desempeñaba una función de atención básica donde trabajaban un técnico paramédico y un conductor de vehículo (para transportar hacia la ciudad de Arica los pacientes más graves), quien prestaba servicio de ayuda. Cada mes llegaba el médico general de zona de Arica, una enfermera, un odontólogo y un asistente social, quienes atendían a los pacientes durante todo un día para luego volver hacia la ciudad. El proceso de cambio se dio cuando hizo falta la presencia fija de algunos profesionales para garantizar la continuidad de asistencia a los pacientes. Así se amplió el grupo de profesionales, se mejoró la infraestructura del consultorio y se planificó una atención médica más estable con un enfoque de trabajo familiar intercultural. Entre 2001 y 2004 cuando se emprendió la cogestión entre el PESPI y la administración municipal sobre asuntos de salud, los profesionales pudieron empezar a manejar fondos dirigidos a colmar las ineficiencias de la estructura de atención primaria:

\section{"Nos preguntábamos que quería hacer el PESPI con estos recursos del Servicio de Salud; ¿era su objetivo beneficiar a la población? El PESPI en lugar de proponer una forma para financiar cosas que más faltaban implementó el diálogo social para llegar al modelo de salud intercultural ${ }^{5 "}$.}

La gestión a nivel municipal y la gestión estatal a través del Servicio de Salud de Arica (el PESPI hace parte del mismo aparato) llevan consigo contradicciones de las que depende la gestión ambigua de la salud de los pacientes aymara.

De hecho los objetivos que un consultorio tiene que lograr para acreditarse como CESFAM se articulan dentro del campo político. La organización del centro debería tener una perspectiva de gestión familiar de los problemas de la comunidad. Los profesionales han de tener cierta continuidad con respecto de su cargo y no ser reemplazados cada tres o seis meses, que es lo que pasa comúnmente para los honorarios que trabajan un tiempo limitado tras haber sido nombrados directamente por el alcalde. La presión por demostrar el logro de dichas metas genera frustración entre la plantilla del centro, con la consecuente insatisfacción en llevar a cabo el trabajo de ayuda. Todo esto se traduce en el abandono de los pacientes, que frente al cambio de operadores, pierden confianza en el sistema de atención, ya que esta fórmula no favorece la creación de relaciones de empatía, que en cambio resultan ser fundamentales para ambas partes.

Desde cuando se empezó a concebir el consultorio como centro de salud familiar se vino organizando la atención de salud cada vez más en términos interculturales: así que la presencia de los médicos aymara en el consultorio representó la máxima expresión del nuevo estándar adoptado por la municipalidad. Los profesionales más expertos han venido manejando las dinámicas entre el centro médico y la comunidad ${ }^{6}$.

Los actores institucionales establecen cómo el centro tiene que gestionar la salud de la población transformando la dimensión del cuidado en algo muy politizado, donde la medicalización es el efecto más directo, y el control de la vida del sujeto aymara parece ser la consecuencia más evidente.

Sobre todo en el caso de pacientes con dolor la medicalización y control se articulan en las prácticas biomédicas por parte de los profesionales que tienen que responder a lógicas planificadas desde un punto de vista institucional.

Más concretamente el saber biomédico tiene la predisposición a sanar los pacientes con dolor enfocándose en la sanación de la parte afectada, localizando y tratando el dolor particular. El suministro de "paracetamol para todos" en los casos de dolor genera una insatisfacción de los pacientes que piden otro tipo de atención. Además los profesionales viven una frustración laboral porque, en la mayoría de los casos por falta de tiempo o dependiendo de las lógicas políticas que establecen las pautas de atención, no pueden ejercer la profesión orientando el proceso terapéutico hacia el apoyo a la persona. Esta situación conlleva una transformación del médico general de zona en una suerte de "entregador 
de fármacos": en la mayoría de los casos se trata de fármacos inadecuados a disminuir el dolor agudo de la persona que sufre. El poder simbólico que se atribuye al medicamento perpetra un proceso de medicalización según el cual la inducción a ese tipo de fármaco -cuya eficacia resulta ser mínima- no se sustituye a la oportunidad, para el paciente, de tener acceso a fármacos más específicos que no se pueden hallar en el consultorio.

En muchos casos de pacientes entrevistados, que lamentan dolores causados por enfermedades crónicas, pero también por problemas osteoarticulares o patologías respiratorias graves, el suministro de antidoloríficos no resulta suficiente como para satisfacer las necesidades del paciente: él no tiene acceso a terapias farmacológicas específicas más adecuadas, amén de no poder compartir la construcción de significados sobre el dolor que vive; dicho proceso de construcción se da a través del diálogo con los profesionales de salud, en cuanto negociación entre seres humanos que permite manejarlo, nombrarlo e incluirlo nuevamente en la cotidianidad del sujeto que padece.

Los límites de una práctica médica que no incluya una comunicación posible, muchas veces por falta de tiempo, de preparación de los profesionales, o por la mala gestión de las relaciones laborales en el equipo, impiden la negociación necesaria para que se cumpla el proceso de cura. En este sentido la relación médico-paciente representa algo fundamental para que se dé una mejora del estado de salud: esto en la mayoría de los casos de dolor no pasa por la eliminación de los síntomas.

Los terapeutas aymaras, aun reconociendo los límites de sus prácticas en la gestión de dolor crónico, sin embargo permiten al paciente -con quien comparten significados sobre la enfermedad y el dolor-incluir la escucha en la dimensión existencial de la persona, acompañando el cuidado con los masajes tradicionales y el suministro de infusiones de hierbas medicinales. La continuidad en la relación terapéutica consiente compartir y manejar juntos la condición de padecimiento así como la construcción de significados comunes para el proceso de sanación. Esta se transforma en la conciencia, por parte del enfermo, de la mejora de su estado, percibido como algo dirigido a su bienestar y que, desde luego, adquiere cierta eficacia real.

La validez de la terapia no responde a criterios propios de la biomedicina, sino a cómo el paciente percibe el manejo de su condición dramática por parte de sus terapeutas. Por esto aquí ha sido fundamental explicar cómo están estructuradas las políticas de gestión de dolor en el campo de la salud intercultural, antes de profundizar el punto de vista de los mismos pacientes entrevistados y apoyándonos en la etnografía.

\section{Gestión de dolor en salud intercultural}

La cultura, en su manifestaciones, permite al paciente padecer el dolor a través de modelos explicativos y categorías inherentes al grupo. $\mathrm{La}$ paciencia, la posibilidad de acceder a tratamientos para aliviar el dolor, los significados que proceden de su etiología son herramientas que el paciente usa para gestionar su experiencia fuera del juicio moral que define el sufrimiento en relación con el pecado.

El cuerpo es el lugar en donde el sujeto experimenta la percepción del dolor, donde las facultades y capacidades de la persona para enfrentarlo encarnan un drama siempre actual: la incorporación del sufrimiento se determina entre la percepción de un dolor siempre vivido históricamente y manejado dentro de la cultura de uno.

En todos los contextos de intervención la práctica biomédica suele reprimir el dolor, eliminándolo a través del suministro farmacológico, incluso cuando no se puede, olvidando lo que esta situación genera a nivel íntimo en la vida del paciente y de su entorno familiar. En el consultorio de Putre el paciente no tiene el acceso a fármacos específicos que, en el caso de dolor crónico, representan algo fundamental para permitir el desarrollo de una vida digna; en cambio el que padece está constantemente presionado por los profesionales que brindan una solución a través de medicamentos básicos que no tienen ningún efecto de mejora para el paciente.

Además el sentido del dolor, en cuanto incorporación de sufrimiento, no se cultiva dentro de la relación profesional-paciente, perpetrando una violencia hacia quien padece: la condición de dolor que él vive (en su cuerpo y al mismo tiempo en su propia existencia) no se gestiona de una forma adecuada como para mejorar su vida. En el centro médico, entonces, esta manera de hacerse cargo del dolor le restituye al paciente esa falta de reconocimiento respecto de la existencia misma de su dolor, lo cual acaba teniendo efectos yatrógenos:

"Yatrogenía es clínica cuando el dolor, la enfermedad y la muerte dependen de la 
atención médica; es social cuando las políticas sanitarias fortifican una organización industrial que genera malestar; es cultural y simbólica cuando una conducta o varias ilusiones originadas por la medicina recortan la autonomía de las personas insidiando sus capacidades de crecer, de cuidarse el uno del otro y de envejecer, o cuando la intervención médica mutila la posibilidad personal de manejar el dolor, la invalidez, la angustia y la muerte (Illich, 1976: 281, trad. mía)".

El paciente experimentando el dolor en su propio cuerpo, en la exteriorización de su condición, reafirma su capacidad de existir en el mundo y dentro de la historia, en cuanto cuerpo lacerado y vivo; la localización de dolor en su propio cuerpo le permite hablar incluyéndolo en una red relacional hecha de nuevas figuras profesionales involucradas en el control de la vida del paciente indígena; demostración esta de la existencia de una marcada dimensión biopolítica en este ámbito intercultural.

El paciente que a través de su cuerpo, su lenguaje y su gestualidad más instintiva, reivindica su propia condición de sufrimiento, reconquista un espacio que es lo del diálogo para ser escuchado, reconocido; no solamente escuchar su cuerpo, sino también su historia: cuerpo, voz, gestualidad definen nuevamente una identidad fragmentada en el tiempo seguidamente a la subalternidad de este pueblo.

La condición existencial de la persona que sufre es la de la destrucción de su propio mundo; la invalidez que el dolor provoca en el paciente (sobre todo el dolor articular) genera una medicalización que se da a través del suministro excesivo de analgésicos. Esta tipología de fármacos no sirven a aliviar, suprimir, eliminar los síntomas de dolor padecidos por los habitantes de Putre, aunque la posibilidad de obtenerlos induce a desear una solución definitiva e inmediata. La ilusión que el sistema médico deja a sus pacientes reproduce relaciones de subalternidad; de hecho ellos luchan contra la ineficiencia de las terapias porque estas no incluyen la posibilidad de establecer una narración que les permita una significación compartida.

De lo que hemos argumentado hasta ahora resulta imposible establecer un límite entre la corporeidad, entendida como "presencia en el mundo" -caracterizada por una agentividad que siempre es corporal-y el drama subjetivo de la percepción del dolor vivido a través del cuerpo. Además puesto que la dimensión física se superpone, confundiéndose, con las condiciones materiales de existencia, considero útil recurrir a las entrevistas hechas a raíz de la aserción de la psicóloga del CESFAM de Putre, cuando le preguntaba cómo sus pacientes articulan el discurso sobre el dolor:

P: Acá en Putre mis pacientes casi nunca
me hablan en términos de dolor, en la cor-
dillera los abuelitos sí, los que están con
artrosis se refieren siempre a un malestar
relacionado a sus dolores corporales, mez-
clan las cosas, me hablan de sufrimiento
y dicen: "Señorita me duele tanto". Y me
doy cuenta que cuando quieren hablarme de
un estado emocional se refieren siempre a
tristeza para no poder hacer las cosas como
las hacían antes, cuando eran jóvenes, por
haber perdido alguna destreza. Ellos, sí,
hablan de dolor cuando es físico, y sentirlo
les impide hacer las cosas como antes, sí
que en este caso también cuando hablan
de un malestar se refieren al dolor. Parece
que lo relacionan más con lo físico, con
el cuerpo que no funciona bien y ellos se
sienten que no pueden hacer. Pero cuando
se ponen a conversar, siempre me hablan
de pena, tristeza, sentirse mal. "Dolor"
lo ocupan cuando hay algo que les duele
físicamente y les impide vivir bien?

El aspecto que sobresale de este fragmento parece ser lo que acentúa cómo el paciente en esta zona y sobre todo los pacientes de la cordillera (que el equipo atendía en las rondas médicas ${ }^{8}$ ) recurren a la palabra "dolor" para enfatizar la invalidez que esta condición genera en la posibilidad de llevar su propia vida.

Son las consecuencias que este dolor genera las que más preocupan, sobre todo en relación a la imposibilidad (cuando hay dolor) de hacer actividades cotidianas de subsistencia, como el trabajo, el pastoreo de llamas y todo el quehacer que la dura vida en la cordillera conlleva.

Para los pacientes entrevistados no es suficiente reducir el dolor a algo que se percibe y por esto representa un problema cuando impide el desarrollo de las actividades cotidianas. Desde el momento en que en este artículo no se puede dar cuenta de todo el material etnográfico recogido, prefiero 
presentar fragmentos de entrevistas a pacientes con dolor, unos testimonios muy esclarecedores de las que son las distintas formas en las que el dolor se habla, sana y significa en relación con la atención médica brindada por el CESFAM con el apoyo de los terapeutas aymaras.

Los pacientes siempre les hablan de su enfermedad a los terapeutas aymaras en términos de dolor. Cuando el equipo del consultorio trabaja en las rondas médicas para visitar a los pacientes de la cordillera y precordillera se suele redactar una hoja de resumen ${ }^{9}$ con los problemas de los pacientes atendidos por las q'lliris y por el yatiri del CESFAM. Revisando la hoja rellenada por el facilitador intercultural (y que yo también llené, cuando ayudé en el trabajo en equipo), es evidente que las razones que justifican las visitas, además de las causas relacionadas con un malestar, siempre están representadas en términos de dolor:

Dolor muscular para nervios y estrés, dolor al músculo del brazo derecho, dolor de talón, dolor completo para enfermedad familiar, dolor a los pies de puro frío, dolor a los huesos y mal de aire, dolor de rodillas y nudos.

En el registro donde se recopilan los problemas de los pacientes, los terapeutas indican los remedios y las prácticas de sanación que ellos utilizan para estos casos (que son los que más recurren):

\section{masaje de la cabeza a la cintura con hierbas y alcohol, frotación de la parte con hier- bas, masaje completo y parche de llareta, masaje completo con hierbas y lectura de hojas, masaje con hierbas a los pies y a la cintura, frotación al cuerpo y hierbas para infusión, frotación para sacar los nudos, y parche león.}

Como hemos visto en el primer ejemplo de hoja de resumen, la enfermedad siempre se explica con el término "dolor", que muy a menudo tiende a ser puesto en relación con la parte afectada, aunque no exclusivamente. Además, a la hora de ir a visita los pacientes hacen mención de las que son las causas de su propio malestar. Los elementos que los pacientes les facilitan a los terapeutas son necesarios para que empiece la práctica de atención: de hecho el terapeuta, consciente de cuál es el punto de vista del paciente, sabe, observándolo, tocándolo y escuchándolo, cómo cuidarlo.

L: Uno dice: "Me duele la espalda" y te dan una inyección, en ese momento nos calma, estamos dos tres días, nos calma y después se va desvaneciendo, y vamos lo mismo. Es un calmante, porque el doctor no te pone la mano, no te toca, no entiende qué es el dolor, de dónde llega; de un mal aire, no hay paracetamol que te pueda sanar, un poquito sí, pero vuelve a repetirse el dolor, no sana definitivamente. Nosotros estamos seguros con nuestros remedios, estamos acostumbrados como las personas de acá para tomar nuestros medicamentos y nos pasa. Siempre podemos curar, pero ellos desconfían que los podemos sanar. El doctor nos dice que uno no tiene nada, que no pasa nada, toman la presión, los exámenes a los pulmones, pero dicen que no le duele, pero la persona dice que le duele, y en este sentido uno tiene que ver ${ }^{10}$.

La mayoría de las veces son los terapeutas aymaras los que dirigen la atención sobre "cómo" el paciente vive su dolor. Ellos comparten y manejan conjuntamente la condición de sufrimiento. Las prácticas terapéuticas aymaras consisten en rituales, masajes con resinas, pomadas y hierbas, hechos por las curanderas de la región. El suministro de infusiones de hierbas, y sus efectos cumulativos, alivian del dolor; los gestos de los médicos indígenas, aunque no tengan un efecto inmediato, así como el suministro de analgésicos, se inscriben en el proceso de sanación, lo que se concretiza en una relación terapéutica continuativa.

El hombre desde el pasado intenta comprender la condición de dolor; no solo la tradición aristotélica lo incluía entre las formas particulares de emoción que el hombre vive, sino que la filosofía mecánica lo veía como una sensación producida por el cuerpo (cuerpo visto como machina), así que la condición de dolor estaba mucho más enganchada con la dimensión física.

La antropología médica se documenta entre varias disciplinas para enfrentar el tema del dolor de lo que definimos "anecdótica de dolor", es decir, lo que esta percibido a nivel subjetivo desde la persona que padece. De hecho: "no hay dolor sin sufrimiento, sin un sentido a nivel objetivo 
que traduzca el movimiento entre un fenómeno fisiológico hacia el centro de la consciencia moral del individuo", percibir dolor destroza la identidad, viola el mundo cotidiano, pone en crisis cualquier capacidad de racionalizarlo, "rasga el hombre a sí mismo y lo pone frente sus límites (Le Breton, D. 1995:11)".

Este dolor está vinculado con la posibilidad de transmitirlo a los que cuidan los pacientes, para que ellos sepan acoger su significado más profundo y respetarlo en el momento del encuentro terapéutico.

Las personas con dolor utilizan narraciones para intentar explicarlo, para decir lo que representa, y entre los aymaras del norte de Chile los que viven en zonas rurales, el elemento cultural de significación establece coordenadas para incluirlo y enfrentarlo cada día de una forma desconocida a los profesionales biomédicos.

La práctica médica del CESFAM, por razones estructurales a su propio campo de intervención, pero también por las normas que orientan las acciones de los profesionales de la salud, sana el paciente, su trastorno, de una forma localizada: manejar el dolor significa reprimirlo, este se transforma en una manifestación/expresión de algo que no funciona en el cuerpo y genera sufrimiento.

M: "El dolor hay que suprimirlo, es un tema cultural, de la sociedad occidental, hay que ver el dolor como algo inaceptable, como algo malo, como algo que no debe suceder. Es importante el tema del sufrimiento, porque hay muchos pacientes que somatizan lo que viven" 11 .

Esta visión de uno de los médicos general de zona, que trabaja en el consultorio, comporta una gestión de los casos de dolor exclusivamente a través del suministro farmacológico en exceso. El poder simbólico que se les atribuye a los medicamentos genera una inducción de necesidades, también cuando tomarlos no tenga ninguna eficacia en la mejoría del estado del enfermo; sobre todo porque en este estudio nos referimos a pacientes con dolor crónico, que no pueden obtener el acceso a terapias farmacológicas específicas.

$\mathrm{R}$ : "Cuando me dio la crisis, como no podía aguantar más el dolor, me fui donde el doctor. Lo que pasa es que había ido antes, porque él cuando empecé a lamentar los dolores sin saber qué era, él me dio aspirina, y esa me produjo otra cosa. Tenía que tomar diez diarias, me hizo pésimo al corazón, me sentía tiritona. Entonces después me dio una crisis fuerte. De allí pasó un año, un año de que él me diagnosticó artritis reumatoide crónica, me empezó por un dedo, ahora prácticamente me afecta las muñecas, los pies, todo. Antes no estaba en las manos. El dolor no estaba en las manos, ahora sí, mi enfermedad está acá a veces. El dolor te ataca todo, toda tu persona, te puede doler todo, está generalizado, de repente te puede doler la muñeca, de repente la rodilla, el hombro, el colon, hasta la cervical, no es un asunto que te duele algo no más. Y me hicieron el test de factor reumatoide, es un análisis de la sangre, resultó positivo, así que descubrí que era verdad lo que me estaba pasando. Al principio me enojé, un poco, ahora lo conozco nada más ${ }^{12}$.

Los pacientes entrevistados que lamentan dolores derivados por enfermedades reumáticas, patologías osteoarticulares y respiratorias crónicas, no tienen el acceso a terapias especificas a su condición. Viven frente a la imposibilidad de empezar un diálogo terapéutico que permita restituir sentido a una existencia quebrada en su sentido más íntimo. La inexistencia de una relación verdadera entre médico y paciente impide la negociación de la cura, que resulta ser fundamental en el proceso de sanación: esto no se traduce para nuestros actores con la eliminación definitiva de su padecimiento.

En los casos estudiados la sanación casi nunca deriva del desvanecimiento de dolor, si no que se concretiza en un proceso relacional, donde las etapas permiten al sujeto mejorar la percepción global de su condición. Reconocer el dolor, y quien lo vive, permite que las dinámicas personales y relacionales lleguen a tener alguna eficacia, según criterios distintos de aquellos biomédicos.

Puesto que el dolor no tiene evidencia y está enredado en la subjetividad del paciente: "exige la capacidad de observarlo o la confianza en la palabra del enfermo (Le Breton 1995: 39)"; por esta razón la intervención de los médicos aymara parece ser la más adecuada para el paciente porque les permite la reconfiguración, a través de un sentido compartido desde un punto de vista social, de la identidad lacerada de su presencia: esto pasa cuando 
el paciente necesita que el médico se ponga en la relación entregándose para cuidarlo.

T: "Nosotros no tenemos las herramientas y el conocimiento que tienen los médicos para ver que tenemos adentro en el cuerpo, y tengo confianza en su trabajo, aunque nosotros también tenemos dolor, y para sacarlo del enfermo lo sentimos. Hay curanderos que perdieron su vida para sacarlo cargándose de mala energía al punto de morir $^{13 "}$.

Tenemos que reconocer la importancia de los significados que nuestra comunidad andina comparte y usa desde el momento que: "todas las sociedades humana integran el dolor en una visión del mundo, dándole sentido, valor, esto disminuye la desnudez y muchas veces su intensidad. El dolor está inscrito dentro de una red de causalidad que explica su origen, y sobre todo los actores que hacen parte del mismo grupo, construyen medidas simbólicas y prácticas para enfrentarlo por medio de las distintas medicinas que cada sociedad elabora (Le Breton, D. 1995: 105)".

En este sentido se necesita que las políticas de la interculturalidad favorezcan el encuentro terapéutico como conjunto de prácticas médicas distintas frente el paciente con dolor, que tiene el derecho a un atención donde exista el respeto, y que esto se traduzca en un atención donde las modalidades personales y colectivas de actuar tengan importancia en el espacio de acción; cada intervención se confronta con la posibilidad efectiva de cuidar el dolor con los recursos disponibles, técnicos, farmacológicos y humanos.

El aludir al lenguaje de usutatwa que interpretamos como término que enuncia, quizás, una aceptación del dolor, en la historia de los pacientes se convierte en una toma de conciencia de sus propios cuerpos, que salen de la esfera privada y se proyectan, al igual que todas nuestras historias, en la esfera pública, compartiendo un conocimiento científico, los signos de sus cuerpos, y los significados de sus propias historias personales. Todos nuestros pacientes problematizan sus categorías y definen su presencia en el mundo a través de la capacidad de nombrar, manejar y significar su dolor, afuera de la posibilidad real que la atención médica disponible ejerce sobre ellos, a través de un sistema intercultural que en teoría, y no siempre en las prácticas, trabaja hacia el bienestar del paciente aymara.
Dialogar sobre la gestión de dolor significa mencionar la eficacia terapéutica en la medida en la cual nos referimos al cuerpo, y a su orden simbólico: justo nos hablamos de una simbolización activa que sobre todo los terapeutas aymaras producen mientras tanto que cuiden el paciente, ayudándolo a gestionar su condición. Prácticas que se superponen al suministro árido de antidoloríficos por parte del médico. Estamos hablando también de eficacia simbólica o sea del momento en el cual la relación entre terapeuta y paciente favorezca la acción de intervención dentro del lazo social.

La colaboración entre dispositivo de eficacia, la gestión conjunta de dolor por parte de distintas perspectivas profesionales, representaría un camino posible para disminuir su intensidad con medicamentos adecuados, para limitar su destructividad así que las personas puedan compartirlo con su red social, transformándolo en una realidad experimentable y comunicable, saliendo del estigma personal.

Nos esperamos que la atención médica prometida por el modelo intercultural chileno esté mayormente preparada a manejar el tema del dolor, gracias a la valorización de las diferencias que teoriza, y por esto volverse más idónea para resolver adecuadamente las necesidades de la comunidad. Así podría haber una colaboración fructuosa entre el enfoque clínico, la farmacopea indígena, las prácticas de manipulación del cuerpo de las q'lliris juntas a las del kinesiólogo, para obtener un nivel alto de servicio. Y permitiendo finalmente que los saberes médicos se ocupen de cuidar el dolor del paciente para que él pueda salir del estigma y del drama que este genera en su existencia. Esto dentro de un escenario tan complejo puede mejorar el estado de bienestar de las personas, y desafiar un proceso terapéutico que pueda definirse como tal, ya que madurado y concretizado en cuanto a práctica de integración a través del trabajo en equipo. Esta perspectiva permitiría que las soluciones potenciales de intervención resuelvan una gestión ambigua de la salud del paciente indígena, visto que la instrumentalización del factor cultural en el campo médico genera un espacio de problematicidad, gravando sobre la posibilidad que tienen las instituciones de optimizar su capacidad de intervención, con consecuente incapacidad de consentir el funcionamiento adecuado de los programas interculturales.

Muchas veces el sistema médico responde a estrategias vueltas a fortalecer el control del sujeto indígena a través del manejo de su salud. 
Él experimentando el dolor en su cuerpo y en su misma vida manifiesta la necesidad de reconquistar un espacio, lo del diálogo y de la escucha; no solamente la escucha de su cuerpo, sino de su historia: cuerpo, voz y gestos definen de nuevo una identidad quebrada en el tiempo de la subalternidad de la población aymara en Chile.

El tema del dolor, como lo de la salud esta absorbida por la cultura médica, por esto resulta fundamental que en este campo específico lleguen actores heterogéneos para incluir significados útiles al paciente: "la réplica de la cultura, es decir, de un horizonte de sentido y actos compartidos, es una instancia antropológica para preservar la complejidad de la identidad y el control de la situación (Le Breton 1995: 164)".

La invalidez que el dolor causa entre los pacientes, la medicalización de la comunidad, inducen al paciente a desear una solución inmediata, y al mismo tiempo a chocar con la ineficacia de un sistema donde las lógicas reconocen el derecho a su salud sin crear las condiciones necesarias para ejercerlo, generando más aún un vacío de significados.

R: "Cada enfermedad tiene su forma de proceder, las reumáticas crónicas, como la mía, generan un dolor inmenso y las personas piensan que esto es la causa de una invalidez necesaria. Yo me muevo, y siento dolor, y sigo en la terapia específica en la ciudad. Los médicos acá son jóvenes, prescriben paracetamol, quizás no saben que no calma tampoco el dolor que tengo. No ando al consultorio, no tomo los fármacos que me dan ellos, y no soy inválida como sucede a los que piensan que tienen que quedarse inmóviles para no sufrir, hago esfuerzo a veces para moverme y aguanto este dolor que me quema adentro, y que conozco bien desde hace muchos años ${ }^{14 "}$.

La práctica médica sorda a la voz del paciente ejerce una violencia: la condición que él vive -y que no está considerada y manejada adecuadamente-frente a sus ojos representa la imposibilidad del reconocer la existencia de dolor:

J: "hay veces que en el pueblo nadie me cree, me voy al consultorio para hacer controles y el médico me manda donde el técnico paramédico para obtener medicamentos, en general paracetamol, sin decir una palabra sobre cómo me siento. Después me pregunto si tengo algo que no funciona y tomo los medicamentos, aunque no se me pasa, no me deja en paz ${ }^{15}$ ".

Los fragmentos de entrevistas enmarcan la intensidad de la experiencia corporal del dolor, él se pone en los lugares de intimidad de la persona que padece, dejando un vacío que se necesita llenar con la relación. El cuidado y el compartir una laceración por parte del equipo de profesionales de salud disminuiría el sentimiento de dolor que implica la sensación de pasividad. Someterse a la acción de una fuerza que tiene el carácter de la impersonalidad aflige la interioridad del ser humano, inhibiendo cualquier intento de control sobre su propia vida. El paciente aymara con dolor vive una doble subalternidad -la que la práctica biomédica acentúa-, ya que está vinculado a la historia de homologación a la identidad chilena: el proceso violento de chilenización que se impone en la memoria de la comunidad, y actúa en el presente como elemento de disgregación social:

M: "me dicen que va a pasar, que sufro, pero va a pasar, me duele todo, todo, y me da vergüenza, porque si no lo digo no me entienden. La señora F. (q'lliri) si, cuando me frota la espalda habla conmigo, me da alivio, como si me sacara el dolor que tengo. Dicen que tengo la depresión, pero no es así, solo siento un dolor inmenso en el cuerpo, y me siento ahogada, oprimida, llueve y me siento sola. Estoy sola ${ }^{16 "}$.

Las palabras de una paciente en visita cada mes a las rondas médicas -lamentaba dolores en todo el cuerpo, y recibía fármacos del médico para encontrar alivio con la $q$ 'lliri del consultorio- hacen un enfoque sobre el drama del dolor de lo que hablamos, muchas veces atado a historias traumáticas que los pacientes comparten.

Esta mujer golpeada por un relámpago tenía unas cicatrices, hemicráneas y un dolor permanente que localizaba en varias partes del cuerpo, según los momentos.

Después del accidente ella sentía que la comunidad la había alejado, además antes del accidente ella perdió el hermano mayor, asesinado por la dictadura de Pinochet, momento de militarización 
de las fronteras y de reclutamiento de la población aymara en el ejército nacional. La oposición del hombre a ser militar chileno se transformó en una tragedia que representa una herida viva en la vida de la mujer que se abre cada vez que ella siente dolor, incomprensible a los médicos del consultorio.

Su desafección a vivir en la ciudad perpetraba su soledad y alienación:

M: "no puedo irme, siempre me sigue mi pena, el dolor que vivo me acuerda de donde llego, no puedo irme, solamente acá siento que puedo estar ${ }^{17}$ ".

Este dolor parece reconducir a la memoria el peso de su historia, avivando cada instante el recuerdo, donde la posibilidad de ser escuchada, creída y aliviada representan un primer avance hacia la reconquista de su derecho a la autodeterminación.

Resulta difícil para nosotros establecer lo que es el dolor de los que nos hablan los pacientes, cómo comprenderlo, cuáles son las categorías para captarlo; es cierto que lo podemos observar en relación con la atención medica que se ofrece al paciente aymara. El estigma, el drama personal y lo de la red familiar de los que padecen son situaciones que en algunos casos están alimentadas por la ineficiencia del servicio médico intercultural que estamos estudiando, hasta el punto de generar una yatrogenia, de la que hablamos al principio de este ensayo.

Es deseable que un sistema como lo que teoriza el modelo intercultural pueda realizar al máximo el potencial que conlleva, y la posibilidad de coordinar relaciones terapéuticas reales, producida de la integración de saberes distintos y complementarios en la capacidad de enfrentar el dolor: tal acción representaría un primer paso concreto hacia el otro que padece, para hacerse cargo de su cuidado, porque: "las prácticas de cura tienen que ver con la definición de espacios de intimidad, se estructuran en un "cuerpo a cuerpo" [...] La cura es eficaz como proceso de relación, no como éxito de curación (Pizza G 2005: 229-230)".

Si esta es la verdad, y los dolores de muchos de los pacientes entrevistados son insanables, la atención medica tendría que ser la manifestación concreta de dicha política de la interculturalidad que en el campo médico reconoce el derecho fundamental para una mejor salud por el paciente aymara, más aún porque es ciudadano chileno.
El objetivo de un servicio médico plural -es decir, la expresión de prácticas plurales interconectadas frente el proceso de cura más adecuado por el paciente- es lo de garantizar a los ciudadanos la superación de las diferencias entre la salud de las personas indígenas y la que pertenece a los connacionales no aymara, que todavía no está resuelta.

Por esta razón los saberes disponibles tienen que juntarse en el trabajo favorecidos desde una política de equidad: hay que cumplir con la demanda de fármacos específicos, pero también hay que reconocer el otro, y el dolor que lo ataña. Solamente así la salud intercultural puede representar un primer paso para lograr el derecho a la "salud para todos".

La polarización entre prácticas distintas, aquí donde la heterogeneidad de atención permitiría un avance en la atención médica y la superación de los límites de la biomedicina, daña la persona que padece, desde el momento que esta sube además la ilusión de una promesa que no se mantiene.

El dominio biomédico perpetra una acción de destacamento de la trama cultural de dolor para optimizar su capacidad resolutiva técnica, saliendo del deber moral de llenar la existencia de quien padece de significados, a través de los recursos humanos sensibles a su sentido más heterogéneo y contradictorio.

La capacidad de las personas de contestar al sentido de su propio mal, por medio de herramientas que la cultura ofrece, viene meno, dejando el hombre solo, frente a su drama: "esta posición aumenta la demanda médica, pero sube la ansiedad del paciente, que se encuentra sin medios para reconquistar su mal (Le Breton 1995: 165)".

Hablar de los riesgos de transformar la comunidad aymara en un grupo anestesiado significa que: "el dolor que se suprime pierde su característica referencial y genera el horror residuo sin significado, que no pone cuestiones (Illich, I. 1976:158)".

La eliminación del dolor -que en los casos de cronicidad representa una utopía- no puede ser la única respuesta por parte de la misma biomedicina que enfrenta el desafío al padecimiento con débiles herramientas (como por ejemplo los analgésicos básicos), porque: "transforma el hombre en un espectador insensible frente el decaimiento de su ser (Illich, I. 1976:159)".

Comprender estos fenómenos, analizarlos en profundidad nos permite esperar que la política intercultural en salud se pueda concretizar en prácticas de relación intercultural tangibles, hechas por 
modelamientos, integración, donde los elementos que conllevan un significado útil sean vistos como "distintamente indispensables", así que el dolor no represente solo algo que hay que suprimir. Con esto no quiero proponer aquí una visión sádica sin reconocer la necesidad de actuar para disminuir el dolor, pero quiero llegar más allá de esta premisa, en los lugares oscuros a nuestra comprensión, donde la eliminación desesperada del dolor no siempre es la forma mejor de sanar. La esperanza de este análisis es la de enfocarnos sobre la importancia que pertenece a los profesionales de salud, que trabajan en las zonas rurales indígenas, para que tomen en cuenta los límites de la biomedicina evitando dejar al paciente a su destino cuando los medicamentos no tienen eficacia. Hay que empezar de nuevo de este punto, con más recursos posibles, un camino, hechos por contradicciones, palabras, gritos, para acordarnos que: "no tendremos nunca el arte de vivir sin dolor, pero, sí tendremos el arte de sufrir menos si lo hacemos de una forma mejor (Russier, J. 1963: 121)".

\section{Agradecimientos}

A la comunidad de Putre y de las zonas cercanas por cuidarme, acogerme como parte de ellos y demostrarme afecto y colaboración, que me permiten, hoy, escribir sobre ellos, con la esperanza de que esta investigación pueda mejorar su condición de vida. Gracias al equipo de CESFAM para incluirme y respetarme profesionalmente. Gracias al profesor Alberto Díaz Araya por los comentarios a este material etnográfico.

\section{Referencias Citadas}

Bastien, J.

1985 Etnofisiología andina. Evidencia lingüística, metafórica, etiológica y etnofarmacológica para conceptos andinos sobre el cuerpo. New York: Cornell University Press.

Baszanger, I.

1998 Pain physicians. All alike, all different, en M. Berg-A. Mol (eds). Differences in medicine. Unraveling practice, techniques, and body, pp. 119-143. Durham: Duke University Press.

Beneduce, R.

2007 Etnopsichiatria. Sofferenza mentale e alterità fra storia, dominio e cultura. Roma: Carocci.

Boccara Bruno, G.

2007 Etnogubernamentabilidad. La formación del campo de la salud intercultural en Chile. Chungara, Revista de Antropología Chilena, 39, 2: 185-207.

Bourdieu, P.

[2003 (1972) ], Per una teoría della pratica. Con tre studi di etnología Cabila. Raffaello Cortina, Milano.

2001 Il campo politico. Bologna: Il Mulino.

Cattaneo, A.

2009 Le politiche sanitarie globali a trent' anni da Alma Ata, en Salute Globale e aiuto allo sviluppo, Pisa: ETS.

Comelles, J.M.

2004 El renacimiento de lo cultural en el Estado de Bienestar, AM, 17-18: 87-10.

Cozzi, D.

2003 Dolore. AM Rivista della Società Italiana di Antropologia Medica. 15-16: 473-503.

Cozzi, D. (a cargo de).

2012 Le parole dell' antropologia medica. Piccolo Dizionario. Perugia: Morlacchi Editore University Press.

Csordas T.

2003 Incorporazione e fenomenologia culturale. Annuario Antropologia. 3:19-42.
Del Vecchio Good Mary-Jo; Brodwin Paul; Good Byron; Kleinman Arthur (eds.)

1992 Pain as human experience. Anthropological perspectives. Berkeley: University of California Press.

De Martino, E.

1961 La terra del rimorso. Contributo a una storia religiosa del sud. Milano: Il saggiatore.

Devoto, G.

1995 Avviamento all'etimologia italiana. Arnoldo Mondadori: Milano.

Farmer P.

2003 Patologies of power. Berkeley: University of California Press.

Foucault, M.

1998 (1963) Nascita della clinica. Un'archeologia dello sguardo medico. Torino: Einaudi.

García Choque, P. A.

1997 Diccionario Aymara-Castellano y Castellano Aymara: Aru Pirwa. Iquique.

Geertz, C.

1998 (1973) Interpretazione di culture, Il Mulino Biblioteca, Bologna.

Good, Byron J.

1999 (1994) Narrare la malattia. Lo sguardo antropologico sul rapporto medico-paziente. Torino: Edizioni di Comunità.

Illich, I.

2004 (1976) Nemesi medica. L'espropriazione della salute. Milano: Bruno Mondadori.

Instituto Nacional de Estadísticas (INE), (CENSO 2002), Estadísticas Sociales de los Pueblos Indígenas en Chile.

Jackson, J.

1994 Chronic pain and the tension between the body as subject and object, in Thomas J. Csordas (ed.) Embodiment and experience. The existential ground of culture and self, pp. 201-228. Cambridge: Cambridge University Press. 
Kleinman, A.

1988 The illness narratives. Suffering, healing and the human condition. New York: Basic Books.

Le Breton, D.

1995 Antropologia del dolore. Roma: Meltemi.

Martínez Hernadéz, Á.

2000 Rendere visibile l'invisibile. L'antropologia e la trasparenza del potere biomedico, AM, 9-10: 9-34.

Menéndez, E.L.

1996 Struttura sociale e struttura di significato nel processo salute/malattia/cura: il punto di vista dell' attore, AM, 1-2: 111-140.

Antropología Médica: espacios propios. campos de nadie, Nueva antropología, Università Rovira y Virgili, Tarragona (disponible en el curso del MAMSI de la Universidad Rovira y Virgili en el año 2009-2010).

MINEDUC Ministerio de Salud. Norma General Administrativa N $^{\text {}} 16$ sobre "Interculturalidad en los Servicios de Salud". Resolución Exenta Nº 261. Abril, 2006. Chile.

Pavanello, M.

2010 Fare Antropologia: metodi per la ricerca etnografica, Zanichelli, Bologna.

Pizza, G.

2005 Antropologia medica. Saperi, pratiche e politiche del corpo. Roma: Carocci.

Quaranta, I.

2006 Antropologia Medica, Milano: Raffaello Cortina Editore.
Ramírez Hita, S.

2011 Salud Intercultural. Crítica y problematización a partir del contexto boliviano. ISEAT, La Paz.

Russier J.

1963 La Souffrance, Ep, Paris.

Scarry, E.

1990 (1985) La sofferenza del corpo. Bologna: Il Mulino.

Scheper Huges, N. Il sapere incorporato: pensare con il corpo attraverso un' antropologia medica critica In: Borofsky R. (a cargo de), ed. L'antropologia culturale oggi. Meltemi, Roma; 2000 (1994):281-95.

Schirripa, P.

Sistema médico. AM. Rivista della Società Italiana di antropologia medica. 2000; 9-10:209-22.

Seppilli, T.

1989 Le tradizioni popolari in Italia. Medicine e magie. Milano: Electa

Servicio de Salud Arica

2006 Perfil Epidemiológico básico de la población aymara. Arica, Chile.

Subsecretaria de Salud Pública y Redes Asistenciales 2009 Salud y Derechos de los Pueblos Indígenas en Chile, 2009. Chile.

Van Kessel, J.

2003 Holocausto al Progreso, los Aymara de Tarapacá. Chile IECTA Iquique.

\section{Notas}

1 El sabio aymara médico del alma y del espíritu.

2 De la entrevista del 3 de enero 2012 con Gumercindo Gutiérrez.

3 Perfil Epidemiológico básico de la población aymara, p.14. (2006). Servicio de Salud Arica

4 Los derechos de los pueblos indígenas explicados para todas y para todos, UNICEF, (2008). Buenos Aires.

5 De la entrevista con el ex director del Consultorio General Aldo Rivera Gahona del 20 de enero 2012.

6 De la entrevista con el ex director del PESPI del 17 de enero 2012.

7 De la entrevista con la psicóloga del CESFAM del 5 de enero 2012.
$8 \quad$ Visitas de atención medica en las localidades de pre cordillera y cordillera de la comuna de Putre.

9 Hoja de resumen de atenciones de médicos aymara del CESFAM.

10 De la entrevista con la q'lliri L. Del 2 de febrero 2012.

11 De la entrevista con el doctor M. Del 24 de noviembre 2011.

12 De la entrevista con la paciente del 5 de octubre 2011.

13 De la entrevista con el Yatiri de Putre del 21 de enero 2012.

14 De la entrevista a una paciente con dolor cronico del 12 de diciembre 2011.

15 De la entrevista a un paciente con dolor cronico del 3 enero 2012.

16 De la entrevista de una paciente del 19 de noviembre 2011.

17 De la entrevista de una paciente del 19 de noviembre 2011. 
